\title{
Investigation of the Effect of Noise Correlations on Diversity Gains and Capacities of Multiport Antennas Using Reverberation Chamber
}

\author{
Xiaoming Chen and Jian Yang \\ Department of Signals and Systems, Chalmers University of Technology, 41296 Gothenburg, Sweden \\ Correspondence should be addressed to Xiaoming Chen, xiaoming.chen@chalmers.se \\ Received 4 July 2012; Revised 27 October 2012; Accepted 5 November 2012 \\ Academic Editor: Min Zhang
}

Copyright ( $) 2012$ X. Chen and J. Yang. This is an open access article distributed under the Creative Commons Attribution License, which permits unrestricted use, distribution, and reproduction in any medium, provided the original work is properly cited.

\begin{abstract}
Most of previous studies on diversity gains and capacities of multiantenna systems assumed independent and identically distributed (i.i.d.) Gaussian noises. There are a few studies about the noise correlation effects on diversity gains or MIMO capacities, however, by simulations only. In this paper, the maximum ratio combining (MRC) diversity gain and multiple-input multiple-output (MIMO) capacity including correlated noises are presented. Based on the derived formulas, measurements in a reverberation chamber are performed for the first time to observe the effect of noise correlations on diversity gains and MIMO capacities.
\end{abstract}

\section{Introduction}

Diversity techniques are used to mitigate fading effects in wireless multipath environments to offer better communication reliability; the multiple-input multiple-output (MIMO) multiplexing, on the other hand, makes use of the scattering property to provide higher communication data rates. Since both the (spatial) diversity and multiplexing in mobile communications involve multiport antennas, the diversity gain and ergodic MIMO capacity become two common parameters for characterization of multiport antennas. This paper focuses on the maximum ratio combining (MRC) diversity gain and the ergodic MIMO capacity. We assume perfect channel sate information (CSI) at receive side but no CSI at transmit side throughout this paper.

MRC diversity gains and MIMO capacities of multiport antennas have been measured in real-life (outdoor and indoor) multipath environments [1-4]. These studies provide valuable empirical results for various representative environments. However, real-life measurements are usually time-consuming and costly. The diversity gain of a multiport antenna can also be evaluated based on anechoic chamber measurements [5]. However, the full radiation pattern measurement is also time-consuming. As an alternative, the reverberation chamber is getting more and more popularity for MIMO terminal characterization (and even system tests) due to its fast and repeatable measurements [6-14]. A reverberation chamber is basically a large metal cavity with mode-stirrers inside to create a Rayleigh-fading environment [15].

While most of the previous works assumed independent, identically distributed (i.i.d.) noises, it was pointed out in $[16,17]$ that the antenna mutual coupling, apart from affecting spatial correlations of received signals, causes noise correlations which, in turn, affect diversity gains and MIMO capacities. However, these studies were carried out by simulations only, and there is so far no measurement for noise correlation effects on either diversity gains or MIMO capacities.

The main purpose of this paper is to study and observe the effects of correlated noises on diversity gains and MIMO capacities by measurements in a reverberation chamber. Formulations of the diversity gain and MIMO capacity including noise correlations (using the noise prewhitening concept) are given in Section 2. The measurements and results are discussed in Section 3, where great care is exerted in choosing appropriate frequency step and frequency stirring bandwidth in order to have accurate measurement results. This paper 
is particularly useful for over-the-air (OTA) measurements using reverberation chambers.

\section{Theory}

Unlike that in [16] where an open-circuit channel concept was used, in the paper, we deal with composite channels including overall antenna effects (i.e., spatial correlation, mutual coupling, and antenna efficiencies) directly, which are more convenient for measured channels [1-14]. Provided that mutual coupling effects can be included correctly using antenna impedance matrices and with the right channel normalization, the open-circuit channel approach gives the same result as the composite channel (including overall antenna effect) approach [4]. In the paper, the MRC diversity gain is presented using the noise prewhitening concept based on [18]. A similar approach is used in the presentation of the MIMO capacity, yet the formulation is rather simple thanks to the well-known MIMO capacity work [19].

2.1. MRC Diversity Gain. Considering a narrowband Nport diversity antenna in a Rayleigh-fading environment, the input-output relation is

$$
\mathbf{y}=\mathbf{h} x+\mathbf{n}
$$

where $\mathbf{h}$ is the composite fading channel vector including overall antenna effect, $x$ is the complex baseband scalar input signal, $\mathbf{y}$ is the complex baseband output signal, and $\mathbf{n}$ is the noise vector. Without specifications, vectors in this paper (e.g., h, y, n) are column vectors. Note that this composite channel model is almost the same as the opencircuit one except that signals are measured with the antenna ports terminated with matched loads instead of opencircuited. In this exhibition, the mutual coupling effect is implicitly included via the composite channel h. To illustrate the equivalence of the open-circuit and composite channel model, we resort to numerical simulations using both models (see Appendix A).

The covariance matrix of the diversity antenna is

$$
\mathbf{R}=E\left[\mathbf{h h}^{H}\right]
$$

where the superscript $H$ is the Hermitian operator, and $E$ is the expectation operator that is usually approximated by sample mean of channel realizations. Note that in a Rayleigh-fading channel, correlation and covariance are used interchangeably, and that in rich scattering environments $\mathbf{R}$ is nonsingular. The instantaneous MRC output power is

$$
P_{\mathrm{MRC}}=\mathbf{h}^{H} \mathbf{h} .
$$

Previous literature assumed i.i.d. Gaussian noises with unity variance, that is, $\mathbf{n} \sim C N(\mathbf{0}, \mathbf{I})$ where $\mathbf{I}$ denotes identity matrix, so that $P_{\mathrm{MRC}}$ was equal (in value) to the instantaneous signal-to-noise ratio (SNR), denoted as $\gamma$ [18]. However, for compact multiport antennas, the antenna mutual coupling colors the noises in different antenna branches, so that $\mathbf{n} \sim C N\left(\mathbf{0}, \mathbf{R}_{n}\right)$. Therefore, $\mathbf{y}$ needs noise prewhitening prior to further signal processing [20]:

$$
\mathbf{y}^{\prime}=\mathbf{R}_{n}^{-1 / 2} \mathbf{y}=\mathbf{R}_{n}^{-1 / 2} \mathbf{h} x+\mathbf{R}_{n}^{-1 / 2} \mathbf{n},
$$

where $\mathbf{y}^{\prime}$ is the prewhitened output signals together with spatially white (unity variance) noises to be combined by MRC. Therefore, the instantaneous SNR is

$$
\gamma=\left(\mathbf{R}_{n}^{-1 / 2} \mathbf{h}\right)^{H}\left(\mathbf{R}_{n}^{-1 / 2} \mathbf{h}\right)=\mathbf{h}^{H} \mathbf{R}_{n}^{-1} \mathbf{h}
$$

and the covariance matrix of the prewhitened signals is

$$
\mathbf{R}^{\prime}=E\left[\left(\mathbf{R}_{n}^{-1 / 2} \mathbf{h}\right)\left(\mathbf{R}_{n}^{-1 / 2} \mathbf{h}\right)^{H}\right]=\mathbf{R}_{n}^{-1 / 2} \mathbf{R}\left(\mathbf{R}_{n}^{-1 / 2}\right)^{H} .
$$

The characteristic function of the MRC output is [21]

$$
\begin{aligned}
\phi(z) & =E[\exp (j z \gamma)]=\frac{1}{\operatorname{det}\left(\mathbf{I}+z \mathbf{R}^{\prime}\right)} \\
& =\frac{1}{\operatorname{det}\left(\mathbf{I}+z \mathbf{R}_{n}^{-1} \mathbf{R}\right)} .
\end{aligned}
$$

Denote $\lambda_{i}(i=1, \ldots, N)$ as the $i$ th eigenvalue of $\mathbf{R}^{\prime}$ (or equivalently $\mathbf{R}_{n}^{-1} \mathbf{R}$ ). The probability density function (PDF) of $\gamma$ is inverse Fourier transform of $\phi(z)$,

$$
p(\gamma)=\frac{1}{\prod_{i} \lambda_{i}} \sum_{i} \frac{\exp \left(-\gamma / \lambda_{i}\right)}{\prod_{k \neq i}\left(\left(1 / \lambda_{k}\right)-\left(1 / \lambda_{i}\right)\right)} .
$$

The cumulative distribution function (CDF) of $\gamma$ is,

$$
F(\gamma)=1-\sum_{i=1}^{N} \frac{\lambda_{i}^{N-1} \exp \left(-\gamma / \lambda_{i}\right)}{\prod_{k \neq i}^{N}\left(\lambda_{i}-\lambda_{k}\right)} .
$$

For a theoretically ideal case (where a multiport antenna is power-balanced and has zero correlations and no mutual coupling among all branches), all eigenvalues are equal to each other, neither (8) nor (9) is valid anymore due to singularity. For such cases (8) and (9) must be replaced by (10) and (11), respectively [22]

$$
\begin{aligned}
& p(\gamma)=\frac{1}{(N-1) !} \frac{\gamma^{N-1}}{\lambda} \exp \left(-\frac{\gamma}{\lambda}\right), \\
& F(\gamma)=1-\exp \left(-\frac{\gamma}{\lambda}\right) \sum_{i=1}^{N} \frac{(\gamma / \lambda)^{i-1}}{(i-1) !} .
\end{aligned}
$$

Note that the CDF formula (9) is almost identical to the one given in [18] except that $\lambda_{i}$ are eigenvalues of $\mathbf{R}_{n}^{-1} \mathbf{R}$ instead of R. This CDF formula is also only valid for multiport antennas with distinct eigenvalues because of its singularity (when any two eigenvalues are equal). Thus one tends to believe that (9) will result in large numerical error when two eigenvalues are close to each other. However, it is has been shown in [7] that (9) does not have large numerical error when the estimated eigenvalues from measurements are close to each other, and therefore holds for practical measurements. Equation (9) will be used in this paper hereafter. 
The effective diversity gain is defined as the output SNR improvement of a diversity antenna compared with that of an ideal single antenna at $1 \%$ outage probability level [22],

$$
G_{\text {eff }}=\left.\frac{F^{-1}(\gamma)}{F_{\text {ref }}^{-1}(\gamma)}\right|_{1 \%},
$$

where $(\cdot)^{-1}$ denotes functional inversion, $F_{\text {ref }}$ is the $\mathrm{CDF}$ of a single ideal antenna,

$$
F_{\text {ref }}(\gamma)=1-\exp (-\gamma) .
$$

2.2. MIMO Capacity. Considering a narrowband MIMO system with $N_{t}$ transmit antennas and $N_{r}$ receive antennas in a flat Rayleigh-fading environment, the input-output relation can be modeled as

$$
\mathbf{y}=\mathbf{H x}+\mathbf{n},
$$

where $\mathbf{H}$ is complex fading channel matrix including overall antenna effects, and $\mathbf{x}$ is complex baseband transmit signal vector. Similarly, due to mutually coupled noise, the noise prewhitening needs to be performed to $\mathbf{y}$,

$$
\mathbf{y}^{\prime}=\mathbf{R}_{n}^{-1 / 2} \mathbf{y}=\mathbf{R}_{n}^{-1 / 2} \mathbf{H} \mathbf{x}+\mathbf{R}_{n}^{-1 / 2} \mathbf{n},
$$

so that the classical MIMO capacity formula given by [19] holds.

Since there is no CSI at transmit side, the transmit power is assumed to be equally allocated to the $N_{t}$ transmit antennas, the ergodic capacity in this case is [19]

$$
\begin{aligned}
C & =E\left\{\log _{2}\left[\operatorname{det}\left(\mathbf{I}+\frac{\gamma}{N_{t}}\left(\mathbf{R}_{n}^{-1 / 2} \mathbf{H}\right)\left(\mathbf{R}_{n}^{-1 / 2} \mathbf{H}\right)^{H}\right)\right]\right\} \\
& =E\left\{\log _{2}\left[\operatorname{det}\left(\mathbf{I}+\frac{\gamma}{N_{t}} \mathbf{R}_{n}^{-1} \mathbf{H} \mathbf{H}^{H}\right)\right]\right\} .
\end{aligned}
$$

2.3. Mutual Coupling on Noises. The mutual coupling effect on noises was studied in [16] for a MIMO system, which is also applicable for diversity antennas. For the sake of completeness, we briefly include the derivation here. Assuming that each antenna port is terminated separately, the spectral density of total thermal noise matrix is

$$
\mathbf{P}_{n}(f)=\frac{1}{2}\left(\mathbf{Y}_{L}+\mathbf{Y}_{L}^{*}\right) \mathbf{v}_{n} \mathbf{v}_{n}^{H},
$$

where $\mathbf{Y}_{L}$ is the diagonal admittance matrix of the loads and $\mathbf{v}_{n}$ is thermal noise voltage. Based on simple circuit theory, we have

$$
\begin{gathered}
\mathbf{v}_{n} \mathbf{v}_{n}^{H}=2 k T \mathbf{Y}_{A}^{-1}\left(\mathbf{Y}_{A}+\mathbf{Y}_{A}^{*}\right)\left(\mathbf{Y}_{A}^{-1}\right)^{H} \\
\mathbf{Y}_{A}=\mathbf{Y}_{R}+\mathbf{Y}_{L},
\end{gathered}
$$

where $k$ is Boltzmann's constant, $T$ is the absolute temperature, and $\mathbf{Y}_{R}$ is the admittance matrix of the receive antennas. For a narrowband system, the normalized noise covariance matrix is

$$
\mathbf{R}_{n}=\frac{\mathbf{P}_{n}(f)}{P_{n}(f)},
$$

where $P_{n}(f)$ is the noise spectral density of an isolated antenna. Note that the exact values of $k, T$ and actual system bandwidth do not matter for $\mathbf{R}_{n}$ since they are all cancelled out by the normalization (19). In order to illustrate the mutual coupling effect on noise correlation, we resort to numerical simulations presented in Appendix B, where it is shown that the mutual coupling effect on noise correlation is more profound with small antenna separation.

\section{Measurements and Results}

To study noise coupling effects on diversity gains and MIMO capacities, we performed measurements of the so-called Eleven antenna (a wideband log-periodic array working from 2 to $13 \mathrm{GHz}$ as shown in Figure 1) [23], in a reverberation chamber. In this case, the wideband array has to be regarded as many narrowband antennas working at different frequencies. Therefore, the wideband measurement is regarded as many separate narrowband measurements for many virtual narrowband antennas operating at different frequencies.

3.1. Measurement of Channel Samples. The chamber used in this work is the Bluetest HP reverberation chamber with a size of $1.75 \times 1.25 \times 1.8 \mathrm{~m}^{3}$. It has two plate stirrers, a turn-table platform, and three wall antennas (antennas mounted on three orthogonal walls inside the chamber) (see Figure 2). In the measurements, the platform moved stepwisely to 20 positions (equally spaced over one complete platform rotation) and for each platform position the two plates moved step-wisely and simultaneously to 10 positions (equally spaced over the total distances that they could travel). At each stirrer position and for each of the three wall antennas, the vector network analyzer (VNA) performed a frequency sweeping to sample the channel transfer functions over frequency. The VNA used in this work is Agilent E5071C ENA series network analyzer working from $100 \mathrm{kHz}$ to $8.5 \mathrm{GHz}$. We therefore chose a measuring frequency range of $2-8 \mathrm{GHz}$. The frequency step was set to $1 \mathrm{MHz}$ (for a reason that will become clear later). Since the maximum sweep point of this VNA is 1601 , we have to divide the whole frequency band into four subbands, that is, $2-3.5 \mathrm{GHz}, 3.5-$ $5 \mathrm{GHz}, 5-6.5 \mathrm{GHz}$, and $6.5-8 \mathrm{GHz}$.

For diversity measurements, the Eleven antenna is regarded as the diversity antenna under test, and channels corresponding to the three wall antennas are considered as the same random process. Therefore, there are 600 channel samples per frequency point for the diversity evaluation. For capacity measurements, the Eleven antenna is regarded as receive MIMO antenna under test, and the three wall antennas are regarded as three transmit antennas. Therefore, there are 200 MIMO channel samples per frequency point for the capacity evaluation. In both cases, the measured channel samples may not be sufficient to support accurate estimations. A simple way to increase channel samples is to treat channel samples at different frequencies as different channel realizations. This methodology has been used in [2] for real-life multipath measurements. In a reverberation chamber, it is usually referred to as the frequency 

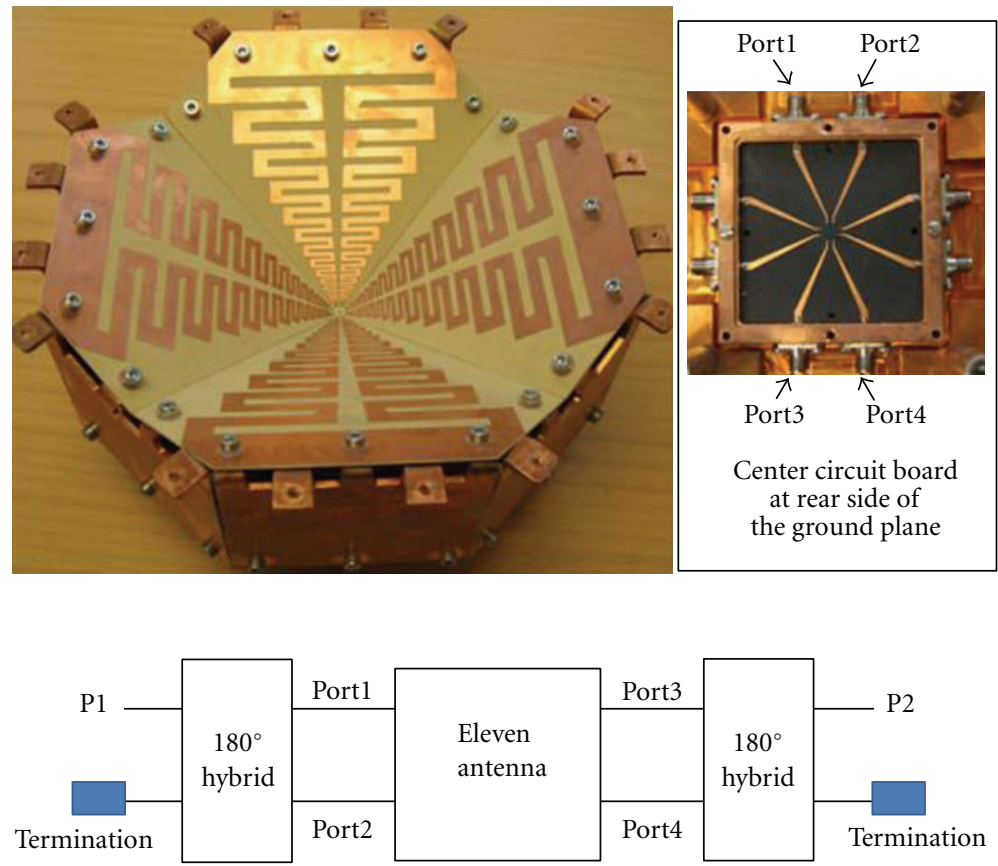

FIGURE 1: Photos of front and back sides of Eleven antenna (upper) and diagram of Eleven antenna with the four ports of one polarization combined to two ports that as used as measurement ports in this paper (lower).

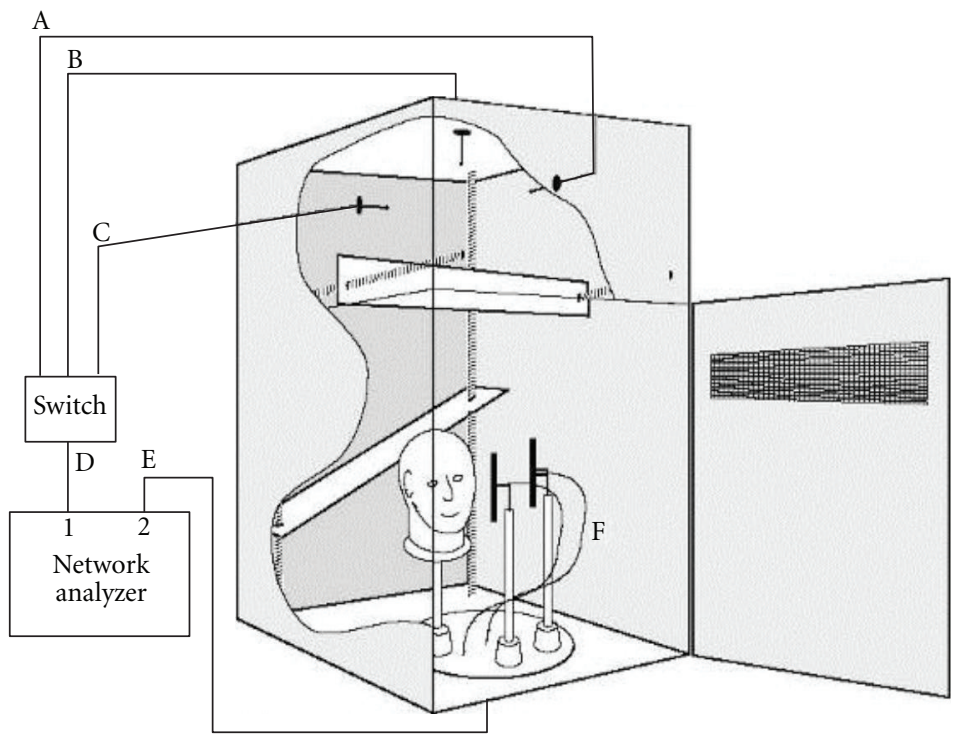

Figure 2: Drawing of Bluetest reverberation chamber measuring a two-port antenna.

stirring or electronic stirring [24]. However, the frequency stirring bandwidth has to be carefully chosen so that more equivalently independent samples can be included without changing the channel statistics. The coherence bandwidth of the channel is around $1-2 \mathrm{MHz}$ [25], while the stationarity bandwidth is larger than $20 \mathrm{MHz}$ (see [8] and the references therein). In practice, the antenna bandwidth also affects the channel characteristics, since the composite channel includes the antennas. Hence, the frequency stirring bandwidth should be larger than coherence bandwidth but smaller than stationarity bandwidth and antenna bandwidth. The Eleven antenna has reflection coefficients below $-10 \mathrm{~dB}$ over its working frequency range [23]. As a result, an empirical frequency stirring bandwidth of $20 \mathrm{MHz}$ is chosen. At this point, the choice of $1-\mathrm{MHz}$ frequency step starts to make sense in that a larger frequency step will degrade the frequency resolution and a smaller one will result in spectrally correlated channel samples (which in turn makes the frequency stirring less effective). As a result, there are 12000 channel samples per frequency for diversity 


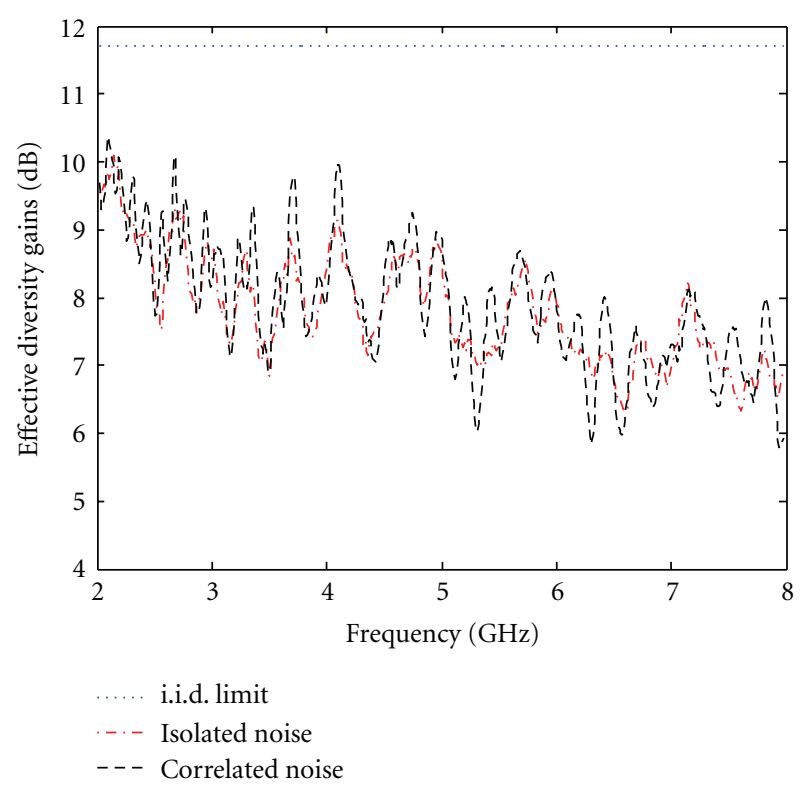

FIgURE 3: Measured effective diversity gains of the two-port Eleven antenna with/without coupled noise.

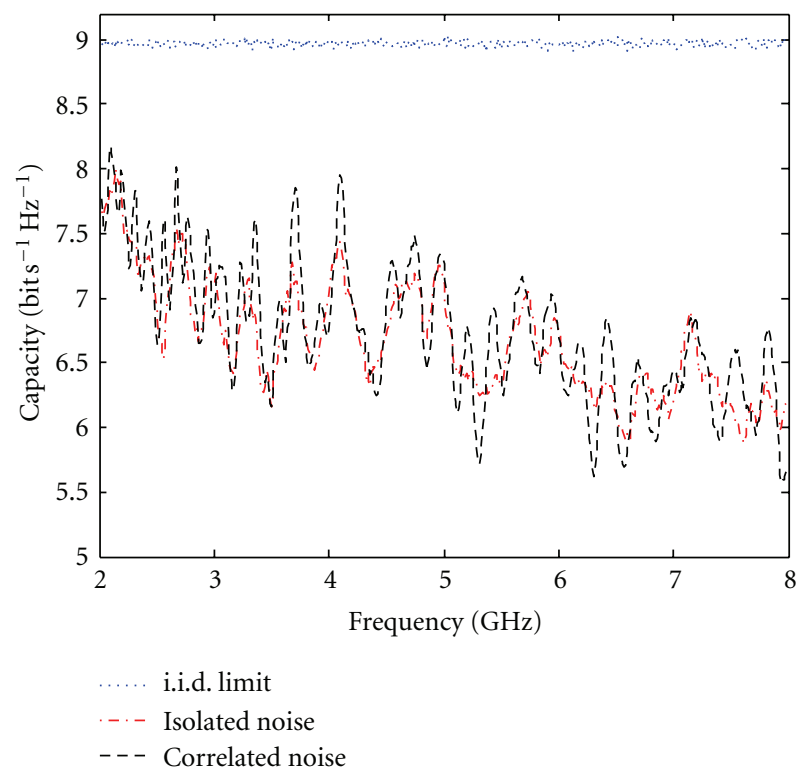

FIgURE 4: Measured ergodic capacities of the two-port Eleven antenna at $15 \mathrm{~dB}$ SNR with/without coupled noise.

evaluations and 4000 channel samples per frequency for capacity evaluations.

In order to calibrate out the long-term fading (or attenuation) in the chamber (so that only short-term fading comes into play), a reference measurement is needed, where the average power transfer function is measured using a reference antenna with known total radiation efficiency. The reference power level, $P_{\text {ref }}$, is obtained by dividing the average power function by the total radiation efficiency of the reference antenna. The measured channel vector $\mathbf{h}_{\text {meas }}$ (for diversity evaluations) and channel matrix $\mathbf{H}_{\text {meas }}$ (for capacity evaluations) are functions of frequency (or virtual narrowband antenna) and stirrer position. For convenience, the normalized measured channel vector and matrix are denoted, respectively, as

$$
\mathbf{h}=\frac{\mathbf{h}_{\text {meas }}}{\sqrt{P_{\text {ref }}}}, \quad \mathbf{H}=\frac{\mathbf{H}_{\text {meas }}}{\sqrt{P_{\text {ref }}}} .
$$

Due to the strong scattering inside the chamber, lineof-sight (LOS) components usually have much smaller power level as scattered components. Therefore, $\mathbf{h}$ and $\mathbf{H}$ in (20) can be considered as zero mean vector and matrix, respectively. Note that the total radiation efficiencies of the wall antennas are also calibrated out by (20). Since the wall antennas are separated sufficiently far from each other (at least 7 8 wavelength spacing at the lowest frequency) and orthogonally polarized, their correlations are negligible [26]. Therefore, the Eleven antenna's effects on diversity gains and MIMO capacities without the effects of the transmit antennas can be examined by the measurement setup and normalization (20) (cf. semicorrelation configurations in [27]).

3.2. Measurement of Correlated Noises. In order to calculate the correlated noises, we have to know the admittance (or impedance) matrix of the Eleven antenna. Note that the derivation in Section 2.3 holds for lossless multiport antennas only. Fortunately, the Eleven antenna itself has a negligible ohmic loss (below $0.3 \mathrm{~dB}$ over the working frequency range) [23]. The $180^{\circ}$ hybrids (see Figure 1), however, have insertion losses between $1.4 \mathrm{~dB}$ at $2 \mathrm{GHz}$ and $3 \mathrm{~dB}$ at $8 \mathrm{GHz}$. In order to calibrate the hybrid losses, we measured S-parameters of the Eleven antenna at the four ports, port1-port4 (see Figure 1) (without the $180^{\circ}$ hybrids) in an anechoic chamber. These measured S-parameters were combined using two ideal lossless $180^{\circ}$ hybrids to obtain Sparameters at "lossless" ports $\mathrm{P}^{\prime}$ ' and $\mathrm{P}^{2}$ '. These "lossless" S-parameters are converted to Y-parameters by

$$
\mathbf{Z}_{R}=Z_{0}\left(\mathbf{I}+\mathbf{S}_{R}\right)\left(\mathbf{I}-\mathbf{S}_{R}\right)^{-1}, \quad \mathbf{Y}_{R}=\mathbf{Z}_{R}^{-1},
$$

where $\mathbf{S}_{R}$ is S-parameter matrix, $\mathbf{Z}_{R}\left(\mathbf{Y}_{R}\right)$ is the impedance (admittance) matrix of the Eleven antenna, and $Z_{0}=$ $50 \mathrm{ohm}$. Assuming 50 -ohm impedance termination at antenna ports, $\mathbf{Y}_{L}=\mathbf{I} / Z_{0}$. Substitute these into (17)-(19), the noise covariance matrix $\mathbf{R}_{n}$ can be calculated.

3.3. Measurement Results. With known $\mathbf{R}_{n}$ and measured channel samples, MRC diversity gains and MIMO ergodic capacities can be readily calculated using the formulas derived in Section 2. Figure 3 shows the measured effective diversity gains with/without noise coupling (i.e., isolated/correlated noise). It is seen that the correlated noises alter the effective diversity gains at some frequencies (corresponding to some virtual narrowband antennas) compared with that of the isolated noise case. Figure 4 shows the measured ergodic capacity with/without noise coupling (i.e., isolated/correlated noise). Similarly, it is found that the correlated noises affect ergodic capacities for some virtual 
narrowband antennas at different frequencies. The ideal diversity gains and ergodic capacities with i.i.d. channel are also plotted, respectively, in Figures 3 and 4 (marked as i.i.d. limits) for comparisons. Note that the i.i.d. limit for ergodic capacities were estimated by the sample mean of mutual information realizations corresponding to 4000 numerically generated i.i.d. Rayleigh-fading channel realizations, while the exact i.i.d. limit for effective diversity gains was calculated analytically using (11)-(13).

There are many factors affecting diversity gain and capacity; however, the "isolated noise" curves in Figures 3 and 4 represent, respectively, the diversity gain and capacity taking into account all the factors except the noise correlation, while the corresponding "correlated noise" curves represent the diversity gain or capacity with all factors including the noise correlation. Therefore, the difference between these two curves is only due to the effect of noise correlation. To clearly illustrate the noise correlation effect on either diversity gain or capacity, we plot the diversity gain and capacity differences (or errors) between "correlated noise" and "isolated noise" cases (in percentage by dividing the differences with the corresponding "correlated noise" value) in Figure 5.

The correlation magnitudes, $\rho$, of the two-port Eleven antenna can be estimated by

$$
\hat{\rho}=\left|[\hat{\mathbf{R}}]_{12}\right|, \quad \hat{\mathbf{R}}=\frac{1}{M N_{t}} \sum_{m=1}^{M} \mathbf{H}_{m} \mathbf{H}_{m}^{H},
$$

where $\mathbf{H}_{m}$ denotes the $m$ th sample of normalized channel $\mathbf{H}, M=4000$ is the number of samples, and $N_{t}=3$ is the number of wall antennas. Figure 6 shows the correlation magnitudes of the virtual narrowband antennas working at different frequencies. Comparing Figure 5 with Figure 6, it is found that diversity gains (and ergodic capacities) with isolated noises are very close to those with correlated noises at frequencies where correlation magnitudes are smaller than 0.2; otherwise, there are noticeable deviations between them and these deviations tend to increase with increasing correlations. In other words, for virtual narrowband multiport antennas with small correlations, it is a good approximation by assuming i.i.d. Gaussian noise, otherwise noise correlations need to be considered in order to get accurate diversity gains and/or ergodic capacities. This observation agrees with the noise power simulation in Appendix B, where it is shown that noise correlation can be neglected when the mutual coupling (or correlation) between the antenna ports is negligible.

This finding verifies the simulation results in [16], where it was shown that the noise correlation has noticeable effect on capacity when parallel half-wavelength dipoles are closer than 0.3 wavelengths, while the parallel halfwavelength dipoles' correlation magnitude is larger than 0.2 when dipole separation is smaller than 0.3 wavelengths in a three-dimensional isotropic-scattering environment [28], for example, a reverberation chamber [29].

\section{Conclusion}

Most of previous diversity and MIMO studies assumed i.i.d. noises in antenna branches. There are only a few works studying the effects of noise correlations on diversity gains and MIMO capacity [16, 17], but these studies were carried out by simulations only. In this paper, the noise correlation effects on diversity gains and capacities are formulated using the noise prewhitening concept and the computational robustness of the derived MRC CDF was proved rigorously. The effects of correlated noises on MRC diversity gains and MIMO ergodic capacities were studied via reverberation chamber measurements, where a great care is exerted in choosing the measurement frequency step and frequency stirring bandwidth in order to have accurate measurement results. It was shown that, only for multiport antennas with very small correlations, the noise correlation effects on diversity gains (capacities) can be neglected. Otherwise, it should be taken into account for accurate diversity (capacity) measurements.

\section{Appendices}

\section{A. Open-Circuit and Composite Channel Model}

From the circuit theory, a MIMO system can be expressed using the open-circuit channel model as [17]

$$
\left[\begin{array}{l}
\mathbf{v}_{T} \\
\mathbf{v}_{R}
\end{array}\right]=\left[\begin{array}{cc}
\mathbf{Z}_{T} & 0 \\
\mathbf{H}^{\mathrm{oc}} & \mathbf{Z}_{R}
\end{array}\right]\left[\begin{array}{l}
\mathbf{i}_{T} \\
\mathbf{i}_{R}
\end{array}\right]
$$

where $\mathbf{Z}_{T}, \mathbf{i}_{T}$, and $\mathbf{v}_{T}$ are impedance matrix, current and voltage vectors at the transmitter, respectively; and $\mathbf{Z}_{R}$, $\mathbf{i}_{R}$, and $\mathbf{v}_{R}$ are impedance matrix, current and voltage vectors at the receiver, respectively; $\mathbf{0}$ is zero matrix with proper dimensions, $\mathbf{H}^{\text {oc }}$ is channel matrix corresponding to open-circuited antennas at both MIMO sides. Note that for notation simplicity and without loss of generality, the additive noises are omitted for the time being, while the noises can be easily included using similar SNR concept as the one used in Section 2.2.1. Based on simple circuit theory, the transmit and receive voltage vectors can be expressed, respectively, as

$$
\mathbf{v}_{T}=\mathbf{Z}_{T}\left(\mathbf{Z}_{T}+\mathbf{Z}_{s}\right)^{-1} \mathbf{v}_{s}, \quad \mathbf{v}_{R}=-\mathbf{Z}_{L} \mathbf{i}_{R}
$$

where $\mathbf{v}_{s}$ is source voltage vector, $\mathbf{Z}_{s}$ and $\mathbf{Z}_{L}$ are source and load impedance matrices, respectively. For coupled impedance matching, both $\mathbf{Z}_{s}$ and $\mathbf{Z}_{L}$ are full matrices, whereas for uncoupled impedance matching, $\mathbf{Z}_{s}$ and $\mathbf{Z}_{L}$ are diagonal matrices. $\mathbf{v}_{R}$ is related to $\mathbf{v}_{T}$ as

$$
\mathbf{v}_{R}=\mathbf{Z}_{L}\left(\mathbf{Z}_{L}+\mathbf{Z}_{R}\right)^{-1} \mathbf{H}^{\mathrm{oc}}\left(\mathbf{Z}_{T}+\mathbf{Z}_{s}\right)^{-1} \mathbf{v}_{s}
$$

The factor $\mathbf{Z}_{L}\left(\mathbf{Z}_{L}+\mathbf{Z}_{R}\right)^{-1} \mathbf{H}^{\text {oc }}\left(\mathbf{Z}_{T}+\mathbf{Z}_{s}\right)^{-1}$ is voltage transfer function. To relate the $Z$-parameter model (A.3) to the information-theoretic input-output relation, $\mathbf{y}=\mathbf{H}_{\mathrm{eff}} \mathbf{X}$, the 


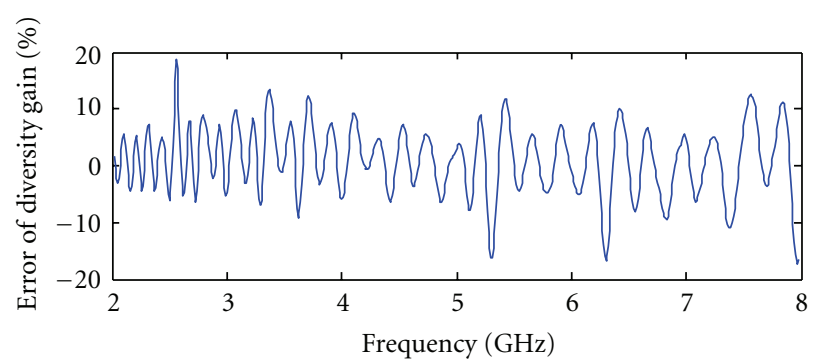

(a)

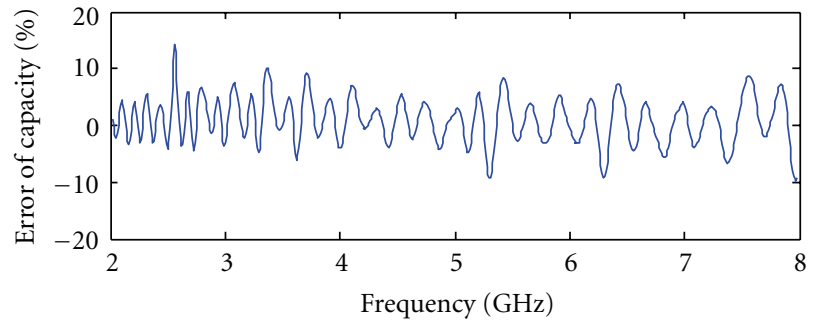

(b)

FIGURE 5: Errors of effective diversity gain and ergodic capacities (at 15-dB SNR) of the two-port Eleven antenna due to noise correlation.

voltage transfer function has to be properly normalized such that the received power satisfies

$$
\begin{aligned}
E\left\{\operatorname{tr}\left[\operatorname{Re}\left(\mathbf{Z}_{L} \mathbf{i}_{\mathbf{R}} \mathbf{i}_{R}^{H}\right)\right]\right\} & =E\left[\operatorname{tr}\left(\mathbf{y} \mathbf{y}^{H}\right)\right] \\
& =E\left[\operatorname{tr}\left(\mathbf{H}_{\text {eff }} \mathbf{K}_{x} \mathbf{H}_{\text {eff }}^{H}\right)\right],
\end{aligned}
$$

where $\mathbf{K}_{x}=\mathbf{I}_{N_{t}} P_{T} / N_{t}$ is covariance matrix of transmit signals. The total radiated power is $P_{T}=E\left\{\operatorname{tr}\left[\operatorname{Re}\left(\mathbf{Z}_{T} \mathbf{i}_{T} \mathbf{i}_{T}^{H}\right)\right]\right\}$. The effective channel can be written as

$$
\mathbf{H}_{\text {eff }}=\sqrt{N_{t}} \operatorname{Re}(\mathbf{Z})_{L}^{1 / 2}\left(\mathbf{Z}_{L}+\mathbf{Z}_{R}\right)^{-1} \mathbf{H}^{\mathrm{oc}} \operatorname{Re}(\mathbf{Z})_{T}^{-1 / 2} .
$$

Accordingly, the effective channel should be normalized to the average channel gain of a SISO system with antennas at both sides conjugate matched, that is, $z_{L}=z_{R}^{*}$ and $z_{s}=z_{T}^{*}$ where superscript $*$ is conjugate operator, $z_{T}$ and $z_{R}$ are antenna transmit and receive impedance, respectively, and $z_{L}$ and $z_{s}$ are load and source impedances at transmit and receive sides, respectively. It is easy to derive the effective SISO channel, that is, $h_{\text {eff }}$, as

$$
h_{\mathrm{eff}}=\sqrt{\frac{N_{t}}{r_{R} r_{T}}} \frac{h}{2}
$$

where $r_{T}=\operatorname{Re}\left\{z_{T}\right\}, r_{R}=\operatorname{Re}\left\{z_{R}\right\}$, and $E\left[|h|^{2}\right]=1$. Dividing $\mathbf{H}_{\text {eff }}$ with $\sqrt{E\left[\left|h_{\text {eff }}\right|^{2}\right]}$, the normalized MIMO channel that includes overall antenna effect is

$$
\mathbf{H}=2 \sqrt{r_{R} r_{T}} \operatorname{real}(\mathbf{Z})_{L}^{1 / 2}\left(\mathbf{Z}_{L}+\mathbf{Z}_{R}\right)^{-1} \mathbf{H}^{\mathrm{oc}} \operatorname{real}(\mathbf{Z})_{T}^{-1 / 2},
$$

where $\mathbf{H}^{\mathrm{oc}}=\boldsymbol{\Phi}_{R}^{\mathrm{oc}, 1 / 2} \mathbf{H}_{w} \boldsymbol{\Phi}_{T}^{\mathrm{oc}, 1 / 2}$, with $\boldsymbol{\Phi}_{R}^{\mathrm{oc}}$ and $\boldsymbol{\Phi}_{R}^{\mathrm{occ}}$ denoting the open-circuit correlation matrix. In order to compare the open-circuit channel model (A.7) with composite channel model, we have to construct the correlation matrix of the signals at the loaded antenna ports (that takes the overall antenna effect, including mutual coupling, into account)

$$
\begin{gathered}
\mathbf{R}=\boldsymbol{\Xi} \circ \boldsymbol{\Phi}, \quad \boldsymbol{\Xi}=\sqrt{\mathbf{e}} \sqrt{\mathbf{e}^{T}}, \\
{[\Phi]_{m n}=\frac{\iint_{4 \pi} \mathbf{g}_{m}^{H}(\Omega) \mathbf{P}_{\mathrm{inc}}(\Omega) \mathbf{g}_{n}(\Omega) d \Omega}{\sqrt{\iint_{4 \pi} \mathbf{g}_{m}^{H}(\Omega) \mathbf{P}_{\mathrm{inc}}(\Omega) \mathbf{g}_{m}(\Omega) d \Omega \cdot \iint_{4 \pi} \mathbf{g}_{n}^{H}(\Omega) \mathbf{P}_{\mathrm{inc}}(\Omega) \mathbf{g}_{n}(\Omega) d \Omega}},}
\end{gathered}
$$

where $\mathbf{g}_{i}(i=1, \ldots, N)$ is the embedded far-field function vector (with elements representing components for different polarizations) at the ith loaded antenna port, and $\mathbf{P}_{\text {inc }}$ is dyadic power angular spectrum of the incident waves, $\mathbf{e}=$ $\left[\begin{array}{llll}e_{\mathrm{emb} 1} & e_{\mathrm{emb} 2} & \ldots & e_{\mathrm{embN}}\end{array}\right]^{T}$, 。 denotes entry-wise product, the superscript ${ }^{T}$ denotes the transpose operator, and $\sqrt{ }$ is entry-wise square root. Note that in a polarization-balanced isotropic reverberation chamber, $\mathbf{P}_{\text {inc }}(\Omega)=$ I. Also note that the transmitting or receiving dependence has been dropped in the expression for notional convenience. The composite channel (including the overall antenna effect) can be expressed as

$$
\mathbf{H}=\mathbf{R}_{R}^{1 / 2} \mathbf{H}_{w} \mathbf{R}_{T}^{1 / 2} .
$$

For simplicity, we use two parallel half-wavelength dipoles as an example. The dipole antennas are used as receive antennas, and two ideal antennas are used at the transmit side. The open-circuit and embedded radiation patterns can be expressed, respectively, as

$$
\begin{aligned}
\vec{G}_{i}(\theta, \phi) & =-\hat{\theta} \frac{2 C_{k} \eta \cos (\pi / 2 \cos \theta)}{k \sin \theta} \exp \left(j k \frac{d_{i}}{2} \sin \theta \sin \phi\right), \\
\vec{G}_{\mathrm{emb}, 1}(\theta, \phi) & =\vec{G}_{1}(\theta, \phi) I_{1}+\vec{G}_{2}(\theta, \phi) I_{2}, \\
\vec{G}_{\mathrm{emb}, 2}(\theta, \phi) & =\vec{G}_{1}(\theta, \phi) I_{2}+\vec{G}_{2}(\theta, \phi) I_{1},
\end{aligned}
$$

where $i=1,2, d_{1}=-d, d_{2}=d, C_{k}=-j k / 4 \pi$, and $\eta$ is the freespace wave impedance. From simple circuit theory, when the excitation current at the port 1 is unity, that is, $I_{1}=1$, 


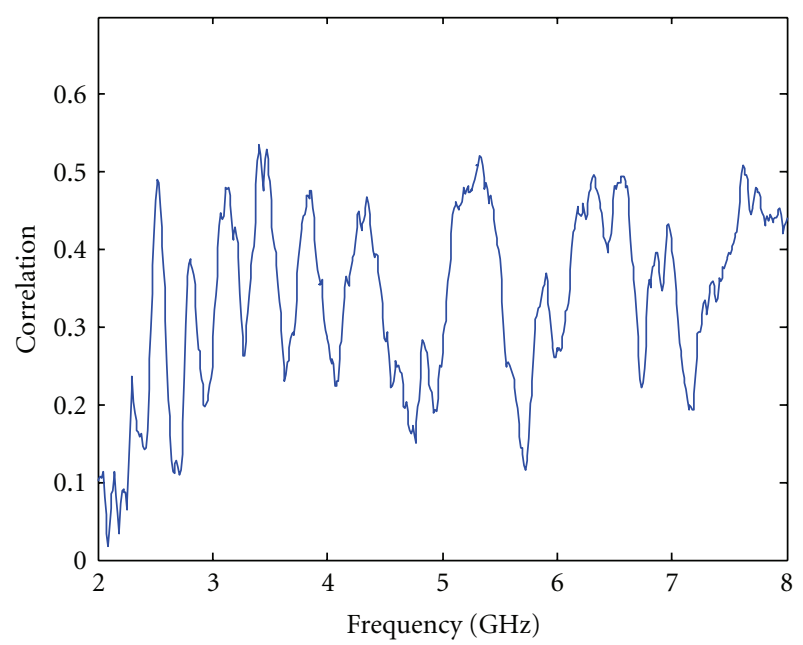

FIGURE 6: Measured magnitudes of complex correlations of the Eleven antenna.

$I_{2}=-Z_{12} /\left(Z_{11}+Z s\right)$. The embedded radiation efficiencies can therefore be calculated as

$$
\begin{gathered}
e_{\mathrm{emb}, j}=\left(1-\left|\frac{z_{\mathrm{in}, j}-z_{L, j j}^{*}}{z_{\mathrm{in}, j}+z_{L, j j}}\right|^{2}\right)\left(1-\frac{r_{L, j j} \sum_{i=1, i \neq j}\left|I_{i}\right|^{2}}{\operatorname{Re}\left\{z_{\mathrm{in}, j}\right\}\left|I_{j}\right|^{2}}\right), \\
z_{\mathrm{in}, j}=z_{j}+\frac{1}{I_{j}} \sum_{i=1, i \neq j} z_{j i} I_{i} .
\end{gathered}
$$

The analytical expressions for the self- and mutualimpedances of the parallel dipoles can be found in [30]. Figure 7 shows the ergodic capacities (as functions of dipole separation) in an isotropic scattering environment at 13dB SNR with 50-ohm loads using both open-circuit and composite channel model. As expected, both models result in the same capacity values.

\section{B. Mutual Coupling Effect on Noise Correlation}

In order to illustrate the mutual coupling effect on noise correlations, we resort to simulations again using the example of two parallel half-wavelength dipoles. As explained in Appendix A, the impedance matrix of the parallel dipoles is given in [30]. Substitute the impedance (or equivalently admittance) matrix into (17)-(19), the noise correlation matrix $\mathbf{R}_{n}$ can be calculated. Since the two dipole antennas are identical, the noise power is simply $\left[\mathbf{R}_{n}\right]_{11}$. Figure 8 calculates the noise power as a function of dipole separation with/without the mutual coupling effect (i.e., correlated noise and isolated noise), where isolated noise is obtained simply by assuming a pair of uncoupled parallel dipoles (with a diagonal impedance matrix). It can be seen from Figure 8 that due to mutual coupling affect the noise power is not white anymore and that it approaches the white noise (i.i.d. noise) asymptotically as dipole separation increases (i.e., as the mutual coupling effect becomes negligible). This observation implies that the noises at different antenna

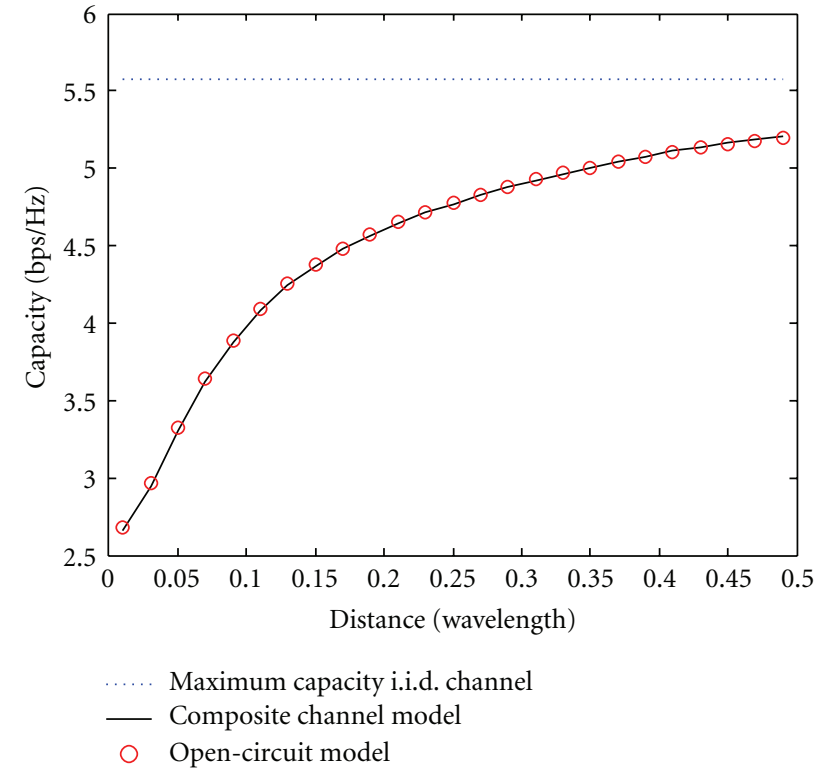

FIGURE 7: Ergodic capacities at 13-dB SNR in an isotropic scattering environment using open-circuit and composite channel models.

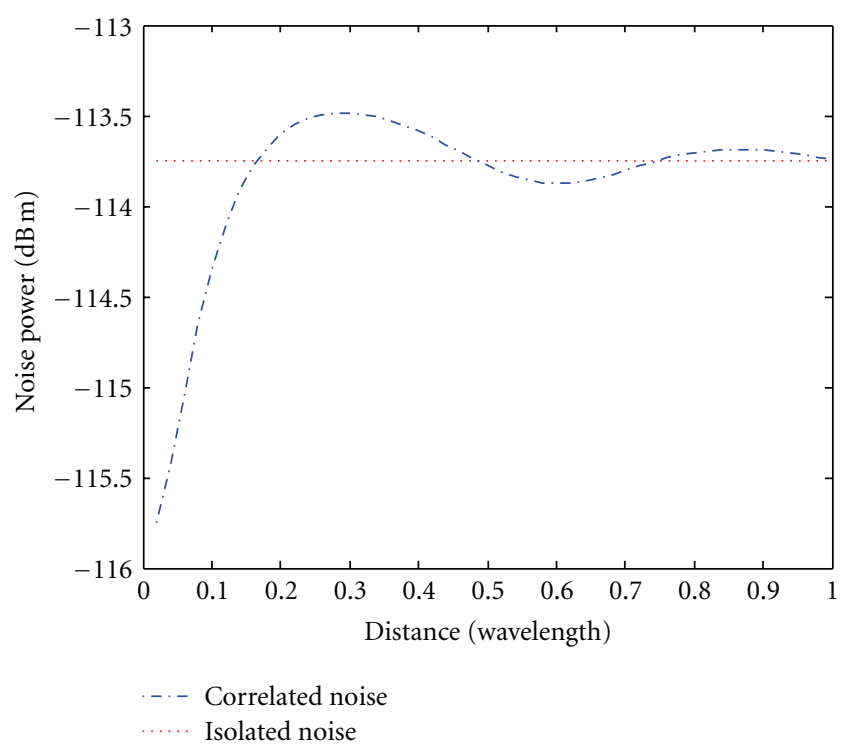

FIGURE 8: Comparison of noise powers of isolated noise and correlated noise.

ports can be approximately treated as uncorrelated when the separation is small.

\section{References}

[1] O. Nørklit, P. D. Teal, and R. G. Vaughan, "Measurement and evaluation of multi-antenna handsets in indoor mobile communication," IEEE Transactions on Antennas and Propagation, vol. 49, no. 3, pp. 429-437, 2001.

[2] K. Yu, M. Bengtsson, B. Ottersten, D. McNamara, P. Karlsson, and M. Beach, "Modeling of wide-band MIMO radio channels 
based on NLoS indoor measurements," IEEE Transactions on Vehicular Technology, vol. 53, no. 3, pp. 655-665, 2004.

[3] A. Diallo, C. Luxey, P. Le Thuc, R. Staraj, and G. Kossiavas, "Diversity performance of multiantenna systems for UMTS cellular phones in different propagation environments," International Journal of Antennas and Propagation, vol. 2008, Article ID 836050, 10 pages, 2008.

[4] Y. Fei, Y. Fan, B. K. Lau, and J. S. Thompson, "Optimal single-port matching impedance for capacity maximization in compact MIMO arrays," IEEE Transactions on Antennas and Propagation, vol. 56, no. 11, pp. 3566-3575, 2008.

[5] V. Plicanic, B. K. Lau, A. Derneryd, and Z. Ying, "Actual diversity performance of a multiband diversity antenna with hand and head effects," IEEE Transactions on Antennas and Propagation, vol. 57, no. 5, pp. 1547-1556, 2009.

[6] K. Rosengren and P.-S. Kildal, "Radiation efficiency, correlation, diversity gain and capacity of a six-monopole antenna array for a MIMO system: theory, simulation and measurement in reverberation chamber," IEE Proceedings, vol. 152, no. 1, pp. 7-16, 2005.

[7] X. Chen, P.-S. Kildal, J. Carlsson, and J. Yang, "MRC diversity and MIMO capacity evaluations of multi-port antennas using reverberation chamber and anechoic chamber," IEEE Transactions on Antennas and Propagation. In press.

[8] X. Chen, "Spatial correlation and ergodic capacity of MIMO channel in reverberation chamber," International Journal of Antennas and Propagation, vol. 2012, Article ID 939104, 7 pages, 2012.

[9] X. Chen, P.-S. Kildal, and J. Carlsson, "Fast converging measurement of MRC diversity gain in reverberation chamber using covariance-eigenvalue approach," IEICE Transactions on Electronics, vol. 94, no. 10, pp. 1657-1660, 2011.

[10] L. Garcia-Garcia, B. Lindmark, N. Jaldén, and C. Orlenius, "MIMO capacity of antenna arrays evaluated using radio channel measurements, reverberation chamber and radiation patterns," IET Microwaves, Antennas and Propagation, vol. 1, no. 6, pp. 1160-1169, 2007.

[11] J. F. Valenzuela-Valdés, M. A. Garcia-Fernandez, A. M. Martinez-Gonzalez, and D. A. Sanchez-Hernandez, "The influence of efficiency on receive diversity and MIMO capacity for Rayleigh-fading channels," IEEE Transactions on Antennas and Propagation, vol. 56, no. 5, pp. 1444-1450, 2008.

[12] K. A. Remley, H. Fielitz, C. L. Holloway, Q. Zhang, Q. $\mathrm{Wu}$, and D. W. Matolak, "Simulation of a MIMO system in a reverberation chamber," in Proceedings of the IEEE EMC Symposium, August 2011.

[13] M. Lienard and P. Degauque, "Simulation of dual array multipath channels using mode-stirred reverberation chambers," Electronics Letters, vol. 40, no. 10, article 578, 2004.

[14] O. Delangre, P. De Doncker, M. Lienard, and P. Degauque, "Coupled reverberation chambers for emulating MIMO channels," Comptes Rendus Physique, vol. 11, no. 1, pp. 30-36, 2010.

[15] J. G. Kostas and B. Boverie, "Statistical model for a modestirred chamber," IEEE Transactions on Electromagnetic Compatibility, vol. 33, no. 4, pp. 366-370, 1991.

[16] S. M. Krusevac, R. A. Kennedy, and P. B. Rapajic, "Effect of signal and noise mutual coupling on MIMO channel capacity," Wireless Personal Communications, vol. 40, no. 3, pp. 317-328, 2007.

[17] M. T. Ivrlač and J. A. Nossek, "Physical modeling of communication systems in information theory," in Proceedings of the IEEE International Symposium on Information Theory (ISIT '09), pp. 2179-2183, Seoul, Korea, 2009.
[18] W. C. Y. Lee, "Mutual coupling effect on maximum-ratio diversity combiners and application to mobile ratio," IEEE Transactions on Communications, vol. 18, no. 6, pp. 779-791, 1970.

[19] G. J. Foschini and M. J. Gans, "On limits of wireless communications in a fading environment when using multiple antennas," Wireless Personal Communications, vol. 6, no. 3, pp. 311-335, 1998.

[20] S. M. Kay, Fundamentals of Statistical Signal Processing: Estimation Theory, Prentice Hall, 1993.

[21] G. L. Turin, "The characteristics function of Hermitian quadratic forms in complex normal variables," Biometrika, vol. 47, pp. 199-201, 1960.

[22] M. Schwartz, W. R. Bennet, and S. Stein McGraw-Hill, Communication Systems and Techniques, 1966.

[23] J. Yang, M. Pantaleev, P. S. Kildal et al., "Cryogenic $213 \mathrm{GHz}$ eleven feed for reflector antennas in future wideband radio telescopes," IEEE Transactions on Antennas and Propagation, vol. 59, no. 6, pp. 1918-1934, 2011.

[24] D. A. Hill, "Electronic mode stirring for reverberation chambers," IEEE Transactions on Electromagnetic Compatibility, vol. 36, no. 4, pp. 294-299, 1994.

[25] X. Chen, P. S. Kildal, C. Orlenius, and J. Carlsson, "Channel sounding of loaded reverberation chamber for over-theair testing of wireless devices: coherence bandwidth versus average mode bandwidth and delay spread," IEEE Antennas and Wireless Propagation Letters, vol. 8, pp. 678-681, 2009.

[26] R. G. Vaughan and J. B. Andersen, "Fading correlations in wireless MIMO communication systems," IEEE Transactions on Vehicular Technology, vol. 36, no. 4, pp. 149-172, 1987.

[27] M. T. Ivrlač, W. Utschick, and J. A. Nossek, "Fading correlations in wireless MIMO communication systems," IEEE Journal on Selected Areas in Communications, vol. 21, no. 5, pp. 819-828, 2003.

[28] X. Chen, P. S. Kildal, and J. Carlsson, "Comparisons of different methods to determine correlation applied to multiport UWB eleven antenna," in Proceedings of the 5th European Conference on Antennas and Propagation (EUCAP '11), pp. 1776-1780, Rome, Italy, April 2011.

[29] D. A. Hill, "Plane wave integral representation for fields in reverberation chambers," IEEE Transactions on Electromagnetic Compatibility, vol. 40, no. 3, pp. 209-217, 1998.

[30] C. A. Balanis, Antenna Theory: Analysis and Design, John Wiley \& Sons, 3rd edition, 2005. 

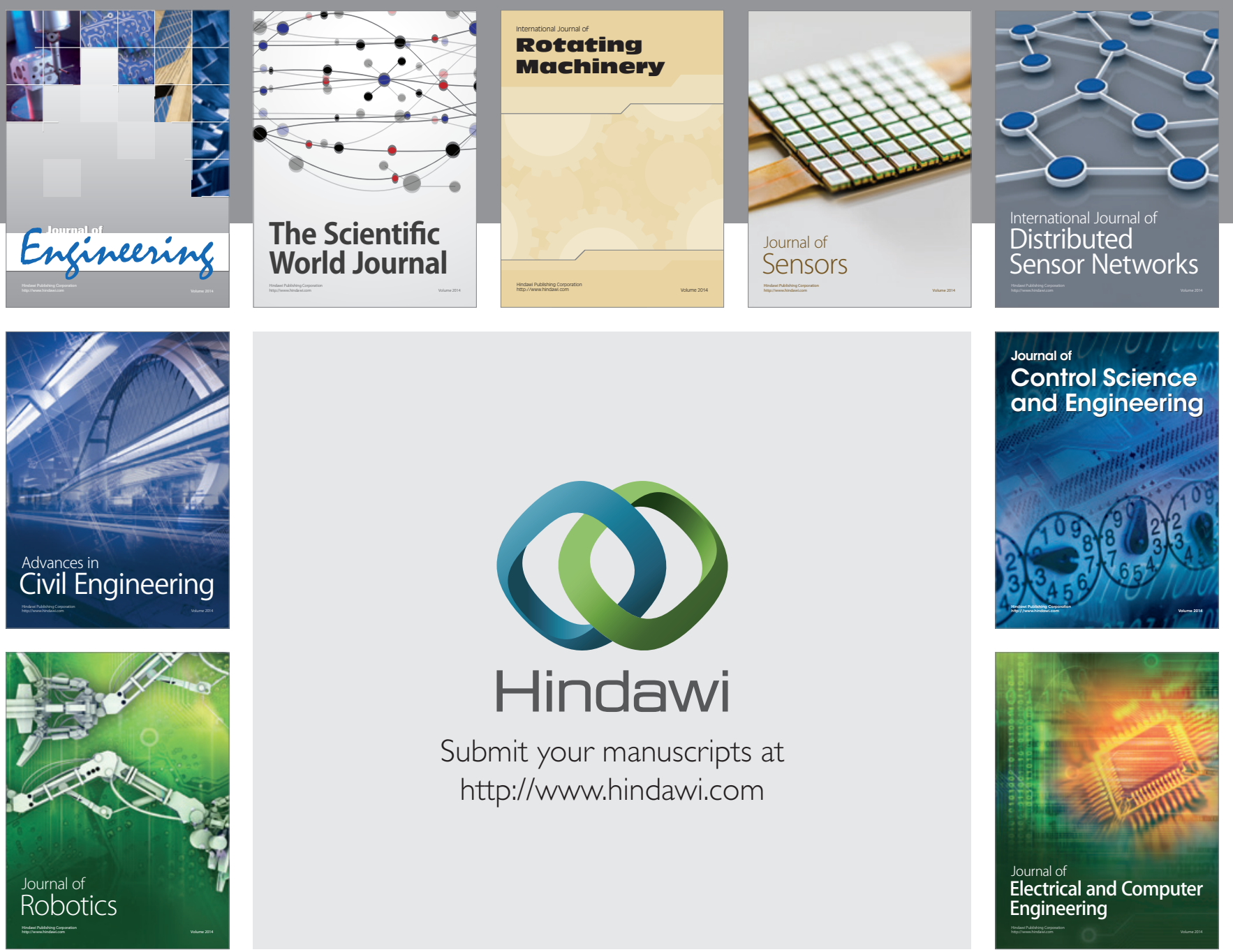

Submit your manuscripts at

http://www.hindawi.com
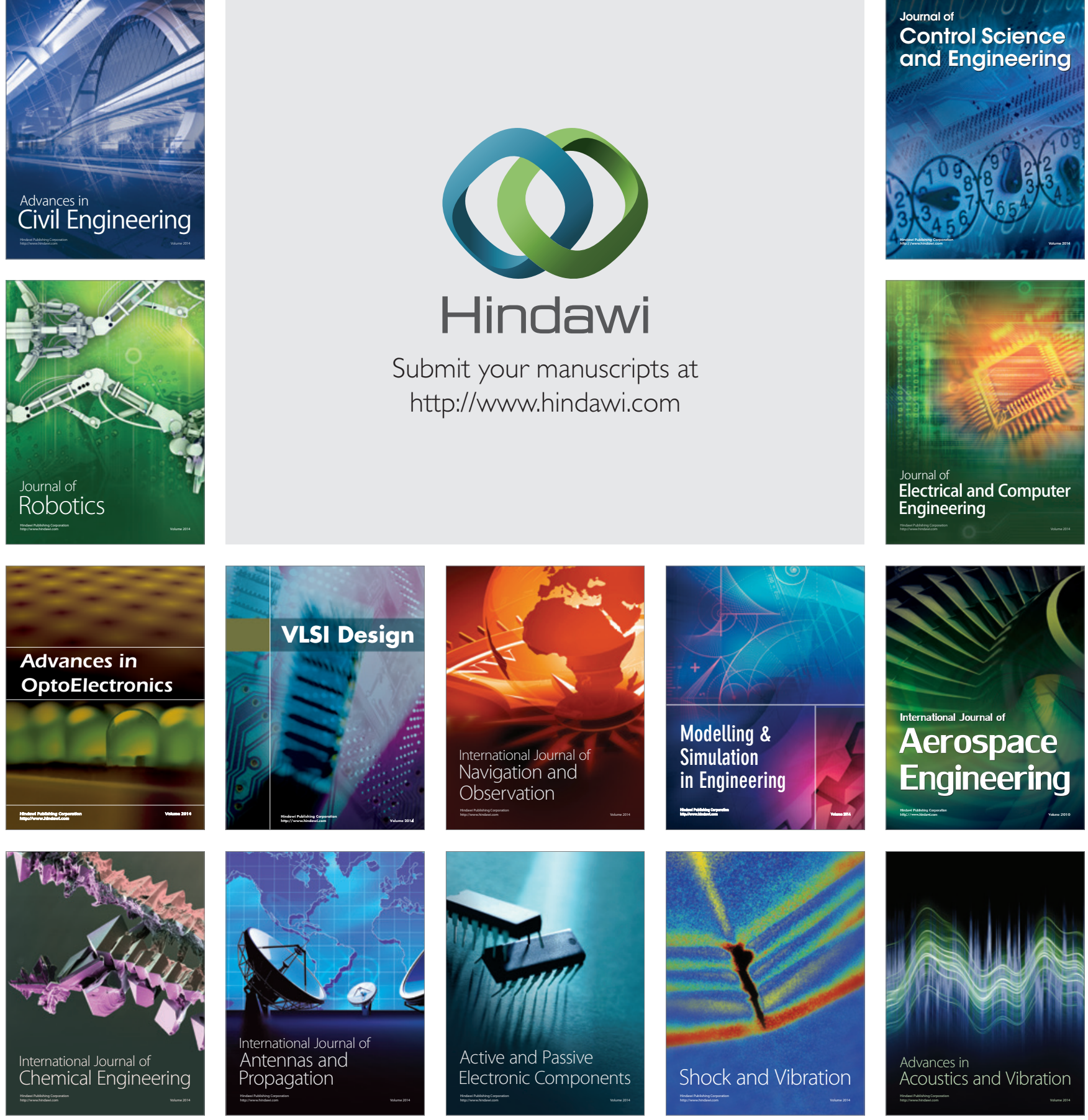